\title{
Comparação entre o teste de despertar e a monitoração neurofisiológica intra-operatória com potencial evocado somato-sensitivo nas cirurgias de escoliose
}

\author{
Comparison between the wake-up test and the \\ intra-operative neurophysiologic monitoring with somato-sensitive \\ evoked potentials in surgery for scoliosis \\ Comparación entre el teste de despertar y la \\ monitorización neurofisiológica intraoperatoria con potencial \\ evocado somato sensorial en las cirugías de escoliosis
}

\author{
Eduardo Barros Puertas' \\ Marcelo Wajchenberg ${ }^{2}$ \\ Ricardo Ferreira ${ }^{2}$ \\ Fabio Fernando Roman Scamardi ${ }^{3}$ \\ Miguel Trandafilov Júnior ${ }^{3}$
}

\section{RESUMO}

Objetivo: comparar a incidência de lesões neurológicas em cirurgias corretivas de curvas rígidas de escoliose idiopática, utilizando-se somente o teste do despertar e utilizando-se a monitoração neurofisiológica intra-operatória por meio dos Potenciais Evocados Somato-Sensitivo (PESS). Métodos: foram realizadas 111 cirurgias para correção de escoliose idiopática, com curvas rígidas por meio de instrumentação e artrodese pela via posterior, no período de janeiro de 1985 a maio de 2001. Os procedimentos foram divididos em dois grupos, sendo 80 pacientes operados sem a monitoração intra-operatória, utilizando somente o teste do despertar, no período de janeiro de 1985 a janeiro de 1998. A partir de maio de 1998, as cirurgias passa-

\begin{abstract}
Objective: the objective of this study is to compare theincidence of neurological injuries in corrective surgeries for rigid curves of idiopathic scoliosis using only the wake-up test and using the intraoperative neurophysiologic monitoring withsomato sensitive evoked potentials. Methods: we performed 111 surgeries to correct idiopathic scoliosis, withrigidcurvesthroughinstrumentation andposteriorfusionintheperiodJanuary 1985 to May 2001. The procedures were divided into two groups, with 80 patients operated without the intra-operative monitoring, using only the wake-up test, from January 1985 to January 1998. From May 1998 the surgeries began to be monitored with somato-sensitive evoked potential (PESS) for prevention of neurological damage, forming a
\end{abstract}

\section{RESUMEN}

Objetivo: comparar la incidencia de lesiones neurológicas en cirugías correctivas de curvas rigidas de escoliosis idiopática, utilizado la prueba del despertar y la monitoración neurofisiológica intraoperatória de por medio de Potenciais Evocados Somato-Sensitivos (PESS). Métodos: fueron ejecutadas 111 cirugías correctivas de escoliosis idiopática rígi$d a$, por medio de instrumentación y artrodesis por vía posterior, entre el período de enero de 1985 ha mayo de 2001. Los procedimientos fueron divididos en dos grupos, siendo 80 pacientes operados sin monitoración intra-operatória, utilizado la prueba del despertar, en el período de enero de 1985 a enero de 1998. Apartir de mayo de 1998 las cirugías comenzaron a ser supervisadas con monitoración delpotencialevocadosomato-sensitivo

\footnotetext{
Trabalho realizado pela Disciplina de Cirurgia da Coluna do Departamento de Ortopedia e Traumatologia da Universidade Federal de São Paulo - UNIFESP - São Paulo (SP), Brasil.

'Professor Associado Responsável pelo Grupo de Patologias da Coluna da Disciplina de Ortopedia do Departamento de Ortopedia e Traumatologia da Universidade Federal de São Paulo - UNIFESP - São Paulo (SP), Brasil.

${ }^{2}$ Médico Assistente Grupo de Patologias da Coluna da Disciplina de Ortopedia do Departamento de Ortopedia e Traumatologia da Universidade Federal de São Paulo - UNIFESP - São Paulo (SP), Brasil.

${ }^{3}$ Médico Estagiário do Grupo de Patologias da Coluna da Disciplina de Ortopedia do Departamento de Ortopedia e Traumatologia da Universidade Federal de São Paulo - UNIFESP - São Paulo (SP), Brasil. 
ram a ser monitoradas com potencial evocado somato-sensitivo (PESS), para prevenção de lesão neurológica, formando um segundo grupo de 31 pacientes. Resultados: no primeiro grupo, um paciente teve lesão neurológica irreversível e quatro pacientes tiveram lesão neurológica reversível. Entre os 31 pacientes do segundo grupo, com monitoração neurofisiológica sensitiva durante a cirurgia, oito apresentaram lesão neurológica reversível constatada no intra-operatório, sem nenhuma repercussão clínica após o procedimento. Conclusão: os resultados apresentados sugerem a eficácia da monitoração intra-operatória, com o potencial evocado somato-sensitivo, para a prevenção de lesões neurológicas, nas cirurgias corretivas de curvas rígidas na escoliose idiopática.

DESCRITORES: Escoliose/ cirurgia; Complicações intra-operatória; Paraplegia/ etiologia; Monitoramento; Potenciais somatosensoriais evocados second group of 31 patients. Results: in the first group, one patient had irreversible neurological damage and fourpatients hadreversibleneurological damage. Among the 31 patients in the second group, with s somato-sensitive evoked potential during surgery, eight had reversible neurological damage in the intra-operatory, without any clinical effect after the procedure. Conclusion: the findings suggest the effectiveness of intra-operative monitoring, with the somato-sensitive evoked potential, for the prevention of neurological injuries in the corrective surgeries of rigid curves in idiopathic scoliosis.

KEYWORDS: Scoliosis/surgery; Intraoperative complications; Paraplegia; Monitoring, Evoked potentials, somatosensory
(PESS) para la prevención de lesión neurológica, formando así un segundo grupo de 31 pacientes. Resultados: en el primer grupo, un paciente presento lesión neurológica irreversible y cuatro pacientes tuvieron lesión neurológica reversible. Entre los 31 pacientes, com monitoración neurofisiológica somato sensitiva, ocho pacientes presentaron lesión neurológica reversible evidenciada enelintra-operatório, sinningunarepercusión clínica después del procedimiento. Conclusión: los actuales resultados sugieren la eficacia de la monitoración intra-operatória, con el potencial evocado somato-sensitivo, para la prevención de lesiones neurológicas, en las cirugías correctivas de curvas rígidas de escoliosis idiopática.

DESCRIPTORES: Escoliosis/ cirugía; Complicaciones intraoperatorias; Paraplejía/ etiologia; Monitoreo; Potenciales evocados somatosensoriales

\section{INTRODUÇÃO}

A cirurgia por via posterior para correção de escoliose é considerada um procedimento agressivo, porém necessária no tratamento das deformidades evolutivas que não são controladas com o tratamento incruento ${ }^{1}$. Várias complicações intra e pós-operatórias são descritas, como alterações cardiovasculares ou pulmonares, hemorragia, lesão peritoneal ou visceral, fratura óssea, infecções, problemas do trato geniturinário e gastrintestina ${ }^{1}$, soltura do material de síntese e pseudartrose $e^{2-4}$. Mas a complicação mais temida é a neurológica, com danos que variam desde alterações leves da sensibilidade até a paraplegia irreversível. A Monitoração Neurofisiológica Intra-Operatória (MNIO) nas cirurgias de coluna vertebral já tem sua eficácia estabelecida na literatura médica mundial como um fator importante na detecção precoce de possíveis lesões neurológicas que possam ocorrer no trans-operatório, prevenindo-se lesões definitivas e suas seqsequela elas, de modo a tornar o ato cirúrgico mais seguro ${ }^{5-8}$.

A proposta deste trabalho é comparar a incidência de lesões neurológicas em cirurgias corretivas de curvas rígidas de escoliose idiopática utilizando somente o teste do despertar e utilizando a monitoração neurofisiológica intra-operatória por meio dos Potenciais Evocados Somato-Sensitivo (PESS).

\section{MÉTODOS}

Foram avaliados 111 pacientes tratados cirurgicamente para a correção da escoliose idiopática por meio de instrumentação e realização de artrodese por via posterior ${ }^{9}$, no período de janeiro de 1985 a maio de 2001. Todas as curvas foram definidas como rígidas ${ }^{10}$, ou seja, com diminuição menor de $40 \%$ da angulação após realizar radiografias com inclinações laterais. Os pacientes foram divididos em dois grupos. O primeiro grupo foi constituído por 80 pacientes, que foram operados utilizando-se somente o teste do despertar em todos os procedimentos cirúrgicos para avaliação intra-operatória de possível lesão neurológica. Neste grupo, a técnica de instrumentação com a haste de Harrington $^{11-13}$ foi aplicada em 38 pacientes, o retângulo de Hartshill, associado com fios de Luque, foi utilizado em 25 pacientes, a haste de Harrington, associada com 
fios de Luque, em 17 pacientes. Todos os pacientes deste grupo foram submetidos à tração halocraniana no período pré-operatório por pelo menos uma semana.

O segundo, foi constituído por 31 pacientes, operados a partir de maio de 1998, utilizando-se a MNIO por meio dos PESS. A técnica de instrumentação com o retângulo de Hartshill foi utilizada em 21 pacientes e a haste de Harrington, associada com fios de Luque, em dez pacientes; 27 pacientes foram submetidos a cirurgia por via anterior para flexibilização da curva.

A MNIO foi realizada por monitoramento continuado comparando-se os resultados obtidos no pré-operatório, pós-indução anestésica e nos pós-operatório. As alterações que preencheram os "critérios de alerta" foram comunicadas imediatamente ao cirurgião, que interrompeu a cirurgia e revisou a técnica cirúrgica no passo anterior à alteração dos potenciais naquele determinado tempo cirúrgico. Os Critérios de Alerta são respostas dos PESS: amplitude com queda $>50 \%$, latência com elevação $>5$ $\mathrm{ms}$, e/ou abolição da resposta. A anestesia, que é um fator importante nas cirurgias com MNIO, foi feita totalmente por meio endovenoso e bombas de infusão contínua, visando manter uma condição mais estável possível do paciente e evitando as grandes variações no grau de consciência e analgesia. As medicações utilizadas de preferência são o Diprivan e remi-fentanil, sem administração concomitante de medicação do tipo inalatória. Parâmetros como pressão arterial média em torno de $65 \mathrm{mmHg}$ e temperatura corporal em torno de $36^{\circ} \mathrm{C}$ também são solicitações feitas aos anestesistas. Os PESS realizados foram de MMSS com estimulação em n. medianos ao nível dos punhos e os de MMII com estimulação de n. tibiais ao nível dos tornozelos na frequencia de $3 /$ seg (Figura 1).

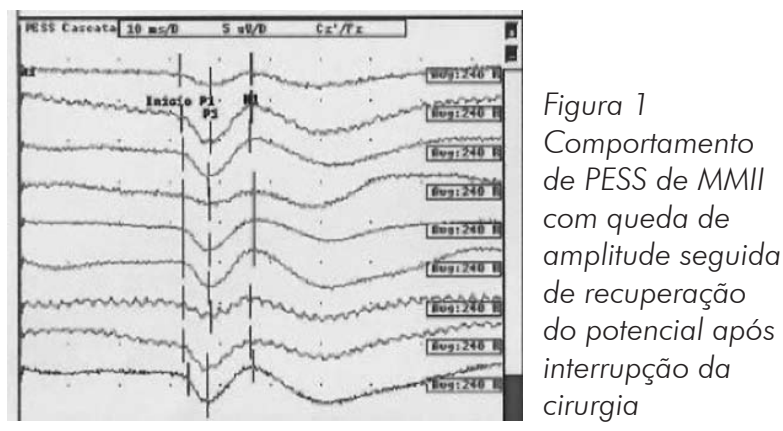

O teste do despertar e monitoração neurofisiológica durante a cirurgia serviram como parâmetros para o diagnóstico e prevenção de lesão neurológica. Toda vez que ambos os testes se mostraram negativos, ou seja, sem resposta motora dos membros inferiores, optou-se pela retirada do implante, realizando-se somente artrodese e imediatamente administravam-se corticóides endovenosos por 24 horas pós-operatórias. Salientamos que na época da realização das cirurgias ainda não havia o potencial evocado motor, e que todas as cirurgias foram realizadas em um serviço de saúde público, apresentando restrições ao uso dos materiais.

\section{RESULTADOS}

Dos 38 pacientes tratados com haste de Harrington, um $(2,64 \%)$ teve lesão neurológica (paraplegia) completamente revertida. Entre os 25 instrumentados com o retângulo de Hartshill, um (4\%) teve alterações neurológicas (paraplegia) irreversíveis, o qual apresentava curva de alto valor angular e extremamente rígida, e outros dois $(8 \%)$ tiveram lesões reversíveis. No grupo dos pacientes instrumentados com a haste de Harrington associada aos fios de Luque ${ }^{14-16}$, notou-se um $(5,88 \%)$ pacientecomalteraçõesneurológicas (paraplegia)reversíveis (Tabela 1). Dos pacientes tratados com auxilio da monitoração neurofisiológica intra-operatória com potencial evocado somato-sensitivo, oito pacientes apresentaram diminuição do PESS intra-operatório com sua recuperação total após um período de pausa e reavaliação dos passos cirúrgicos, sem, no entanto, necessitar a retirada do material. Ao término da cirurgia nenhum paciente apresentou complicações neurológicas (Tabela 2).

\section{TABELA 1 - Pacientes do Grupo 1 submetidos a correção cirúrgica com teste do despertar}

\begin{tabular}{lcc}
\hline Paciente & Técnica empregada & Dano neurológico \\
\hline 1. LOB & Harrington & Não \\
2. VMS & Harrington & Não \\
3. LCCS & Harrington & Não \\
4. TVF & Harrington & Não \\
5. FAR & Harrington & Não \\
6. ASA & Harrington & Não \\
7. SAA & Harrington & Não \\
8.GAA & Harrington & Não \\
9. MCS & Harrington & Não \\
10. DPC & Harrington & Não \\
11. LSS & Harrington & Não \\
12. GSS & Harrington & Não \\
13. APG & Harrington & Não \\
14. NAM & Harrington & Não \\
15. LMS & Harrington & Não \\
16. TPK & Harrington & Não \\
17. VAL & Harrington & Não \\
18. RFM & Harrington & Não \\
19. SMS & Harrington & Não \\
20. AFM & Harrington & Não \\
21. MSS & Harrington & Não \\
22. FDM & Harrington & Não \\
23. DKS & Harrington & Não \\
24. APM & Harrington & Não \\
25. CAAS & Harrington & Não \\
26. SBR & Harrington & Não \\
27. PHMR & Harrington & Não \\
28. ESA & Harrington & Não \\
\hline & & continua...
\end{tabular}




\begin{tabular}{|c|c|c|}
\hline Paciente & Técnica empregada & Dano neurológico \\
\hline 29. KRDR & Harrington & Não \\
\hline 30. LB & Harrington & Não \\
\hline 31. FR & Harrington & Não \\
\hline 32. ESS & Harrington & Não \\
\hline 33. AAF & Harrington & Não \\
\hline 34. EFRF & Harrington & Não \\
\hline 35. AF & Harrington & Não \\
\hline 36. LPO & Harrington & Não \\
\hline 37. SO & Harrington & Não \\
\hline 38. FO & Harrington & Transitória - paraplegia \\
\hline 39. LAS & Hartshill & Não \\
\hline 40. RPS & Hartshill & Não \\
\hline 41. LMB & Hartshill & Não \\
\hline 42. ACSF & Hartshill & Não \\
\hline 43. MAS & Hartshill & Não \\
\hline 44. SFCS & Hartshill & Não \\
\hline 45. RPS & Hartshill & Não \\
\hline 46. ECM & Hartshill & Transitória - paraplegia \\
\hline 47. RTB & Hartshill & Transitória - paraplegia \\
\hline 48. ESA & Hartshill & Não \\
\hline 49. SMC & Hartshill & Não \\
\hline 50. AFF & Hartshill & Não \\
\hline 51. APGM & Hartshill & Transitória - paraplegia \\
\hline 52. $\mathrm{PO}$ & Hartshill & Não \\
\hline 53. DR & Hartshill & Não \\
\hline 54. PAG & Hartshill & Não \\
\hline
\end{tabular}

\begin{tabular}{|c|c|c|}
\hline Paciente & Técnica empregada & Dano neurológico \\
\hline 55. KSA & Hartshill & Não \\
\hline 56. DRG & Hartshill & Não \\
\hline 57. SCG & Hartshill & Não \\
\hline 58. LF & Hartshill & Não \\
\hline 59. MILP & Hartshill & Não \\
\hline 60. TS & Hartshill & Não \\
\hline 61. SJS & Hartshill & Não \\
\hline 62. JAS & Hartshill & Não \\
\hline 63. $C B$ & Hartshill & Não \\
\hline 64. PAG & Harrington + Luque & Não \\
\hline 65. LNCB & Harrington + Luque & Não \\
\hline 66. KSM & Harrington + Luque & Não \\
\hline 67. MFCO & Harrington + Luque & Não \\
\hline 68. IBA & Harrington + Luque & Não \\
\hline 69. OS & Harrington + Luque & Não \\
\hline 70. FS & Harrington + Luque & Não \\
\hline 71. DA & Harrington + Luque & Não \\
\hline 72. SLG & Harrington + Luque & Não \\
\hline 73. EGL & Harrington + Luque & Não \\
\hline 74. MLL & Harrington + Luque & Não \\
\hline 75. LM & Harrington + Luque & Não \\
\hline 76. PRM & Harrington + Luque & Não \\
\hline 77. DR & Harrington + Luque & Não \\
\hline 78. RVM & Harrington + Luque & Não \\
\hline 79. MLMP & Harrington + Luque & Não \\
\hline 80. МТСВ & Harrington + Luque & Não \\
\hline
\end{tabular}

TABELA 2 - Pacientes submetidos a correção cirúrgica com monitoração neurofisiológica intra-operatória e que apresentaram diminuição do PESS com rescuperação sem percussão pós-operatória

\begin{tabular}{|c|c|c|c|c|c|c|c|}
\hline Nome & $\begin{array}{l}\text { Dano } \\
\text { neurológico }\end{array}$ & $\begin{array}{l}\text { Técnica } \\
\text { empregada }\end{array}$ & $\begin{array}{l}\text { Alteração } \\
\text { do PESS }\end{array}$ & Nome & $\begin{array}{l}\text { Dano } \\
\text { neurológico }\end{array}$ & $\begin{array}{l}\text { Técnica } \\
\text { empregada }\end{array}$ & $\begin{array}{l}\text { Alteração } \\
\text { do PESS }\end{array}$ \\
\hline 1. LBR & Não & Harrington + Luque & & 17. JCAS & Não & Hartshill & \\
\hline 2. ME & Não & Harrington + Luque & & 18. GG & Não & Hartshill & Sim \\
\hline 3. EGS & Não & Harrington + Luque & & 19. TS & Não & Hartshill & \\
\hline 4. AK & Não & Harrington + Luque & & 20. CCS & Não & Hartshill & \\
\hline 5. BTG & Não & Harrington + Luque & Sim & 21. GCS & Não & Hartshill & \\
\hline 6. MAG & Não & Harrington + Luque & & 22. LP & Não & Hartshill & Sim \\
\hline 7. FSM & Não & Harrington + Luque & & 23. AAF & Não & Hartshill & \\
\hline 8. CCP & Não & Harrington + Luque & Sim & 24. RAS & Não & Hartshill & Sim \\
\hline 9. EK & Não & Harrington + Luque & Sim & 25. RD & Não & Hartshill & \\
\hline 10. CRB & Não & Harrington + Luque & Sim & 26. FP & Não & Hartshill & \\
\hline 11. FSM & Não & Hartshill & & 27. EG & Não & Hartshill & \\
\hline 12. ISB & Não & Hartshill & & 28. RT & Não & Hartshill & \\
\hline 13. CCB & Não & Hartshill & & 29. EAM & Não & Hartshill & Sim \\
\hline 14. EAM & Não & Hartshill & & 30. FC & Não & Hartshill & \\
\hline 15. JPR & Não & Hartshill & & 31. GAG & Não & Hartshill & \\
\hline 16. CMS & Não & Hartshill & & & & & \\
\hline
\end{tabular}




\section{DISCUSSÃO}

O teste do despertar descrito em 1973 por Vouzelle et al. foi a primeira forma de monitoração do componente motor nas cirurgias de coluna vertebral. É por definição a confirmação visual da movimentação ativa dos pés (MMII), servindo, portanto, para avaliar o paciente do ponto de vista motor. Alguns autores relataram casos em que a monitoração da medula espinhal intra-operatória esteve normal e o teste do despertar demonstrou paraplegia ${ }^{17-19}$.

A MNIO por PESS é a utilização dos testes para observação, durante o trans-operatório, para verificar o funcionamento neurofisiológico das vias ascendente medulares durante as manipulações realizadas em cirurgias de coluna. Os critérios de alerta são respostas do PESS: latência com elevação maior ou igual a $5 \mathrm{~ms}$, Amplitude com queda maior ou iguala $50 \%$ e/ou abolição das respostas. A anestesia ainda tem como fator importante o controle da pressão arterial média, que por si só pode reduzir a amplitude de resposta dos PESS quando esta estiver abaixo dos limites de 60/65 $\mathrm{mmHg}$, provocando falsos positivos.

Durante a utilização de técnicas de distração, todos os tecidos da região são submetidos a intenso torque, inclusive a medula espinhal. Outro fator importante é a diminuição do suprimento sanguíneo para a medula espinhal ${ }^{20,21}$; tal fato foi relatado por Taylor et al. ${ }^{20}$, que discutem o caso que desenvolveu paraplegia no período pós-operatório imediato, após episódio de hipotensão, havendo regressão do quadro neurológico após reposição da volemia.

A abordagem cirúrgica de deformidades rígidas requer cuidados especiais, pois muitos autores indicam que esse é um fator de risco para o desenvolvimento da paraplegia $^{2-4,18,20,22}$. Tais medidas incluem a tração halofemoral no período pré-operatório e a abordagem por via anterior, realizando a discectomia no ápice da curva e em alguns casos até a ressecção do corpo vertebral, conforme relatado por Pedrals ${ }^{23}$.

O trabalho de Cervellati et al., que analisa o tratamento cirúrgico com instrumentação e realização de artrodese por via posterior em 750 pacientes, usando as hastes de Harrington, Hartshill e Luque, mostra alta taxa (4\%) de lesão neurológica permanente nos casos em que foi utilizado o retângulo de Hartshill ${ }^{3}$. Sabemos que o retângulo de Hartshill ${ }^{24,25}$ tem grande rigidez, permitindo elevado torque para correção das deformidades, aumentando os riscos de lesões neurológicas.

Por fim, ressaltamos a importância dos testes do despertar e o potencial evocado somato-sensivitvo no ato intraoperatório, são testes que demonstram sinais de lesão neurológica, permitindo a imediata retirada do material de síntese, conforme demonstrado neste trabalho, no qual oito pacientes apresentaram alteração do PESS sem repercussão clínica no pós-operatório. Vários autores relataram casos em que a monitoração da medula espinhal intra-operatória esteve normal e o teste do despertar demonstrou paraplegia ${ }^{17-19}$. Desta forma, não descartamos o teste do despertar como parâmetro de segurança nas cirurgias da coluna vertebral.

\section{CONCLUSÃO}

A monitoração somato-sensitiva mostrou-se um fator importante na detecção precoce de alterações neurofisiológicas que precedem as lesões neurológicas que podem ocorrer decorrentes do trauma cirúrgico, no momento em que as mesmas ainda possam ser revertidas.

O teste do despertar necessita da cooperação do paciente e a informação pode ser tardia, no entanto, não deve ser descartado como parâmetro da preservação da função neurológica.

\section{REFERÊNCIAS}

1. Schimidt AC. Fundamental principles on treatment of scoliosis. AAOS Instr Course Lect. 16:184, 1959.

2. Carlioz H, Ouaknine M. [Neurologic complications of surgery of the spine in children] Chirurgie. 19941995;120(11):26-30. French.

3. Cervellati S, Bettini N, Bianco T, Parisini P. Neurological complications in segmental spinal instrumentation: analysis of 750 patients. Eur Spine J. 1996;5(3):161-6.

4. Vogel LC, Lubicky JP. Neurologic and vascular complications of scoliosis surgery in patients with Ehlers-Danlos syndrome. A case report. Spine. 1996;21(21):2508-14.

5. Mittal S, Farmer JP, Poulin C, Silver K. Reliability of intraoperative electrophysiological monitoring in selective posterior rhizotomy. J Neurosurg. 2001;95(1):67-75.
6. Logigian EL, Shefner JM, Goumnerova L, Scott RM, Soriano SG, Madsen J. The critical importance of stimulus intensity in intraoperative monitoring for partial dorsal rhizotomy. Muscle Nerve. 1996;19(4):415-22.

7. Maguire J, Wallace S, Madiga R, Leppanen R, Draper V. Evaluation of intrapedicular screw position using intraoperative evoked electromyography. Spine. 1995;20(9):1068-74.

8. Calancie B, Madsen P, Lebwohl N. Stimulus-evoked EMG monitoring during transpedicular lumbosacral spine instrumentation. Initial clinical results. Spine. 1994;19(24):2780-6.

9. Mielke CH, Lonstein JE, Denis F, Vandenbrink K, Winter RB. Surgical treatment of adolescent idiopathic scoliosis. A comparative analysis. J Bone Joint Surg Am. 1989;71(8):1170-7.
10.Kane J, Moe JH. Methods and technique of evaluating idiopathic scoliosis. In: AAOS Symposium on the Spine. St Louis: Mosby; 1969. p. 196-240.

11. Chotigavanich C, Songcharoen $P$, Thanapipatsiri S. Surgical treatment of adolescent idiopathic scoliosis using Harrington distraction rod combined with segmental sublaminar wiring. J Med Assoc Thai. 1994;77(12):617-26.

12.Harrington PR. Spinal instrumentation for management of scoliosis. J Bone Joint Surg. 1960; 42:1448.

13. Harrington PR. Treatment of scoliosis. Correction and internal fixation by spine instrumentation. J Bone Joint Surg Am. 1962;44-A:591-610.

14.Kerboul B, Courtois B. [Segmental posterior spinal osteosynthesis using the Luque-Dove technic] J Chir (Paris). 1989 Mar;126(3):193-8. French. 
15.Luque ER. The anatomic basis and development of segmental spinal instrumentation. Spine. 1982;7(3):256-9.

16.Luque ER. Segmental spinal instrumentation for correction of scoliosis. Clin Orthop Relat Res. 1982;(163):192-8.

17. Ginsburg HH, Shetter AG, Raudzens PA. Postoperative paraplegia with preserved intraoperative somatosensory evoked potentials. Case report. J Neurosurg. 1985;63(2):296-300.

18.Letts RM, Hollenberg C. Delayed paresis following spinal fusion with Harrington instrumentation. Clin Orthop Relat Res. 1977;(125):45-8.
19.Schimidt EW. Post-instrumentation paraplegia and a negative Stagnara test - A case of report [abstract]. In: Annual meeting of the Scoliosis Research Society; 1978 Sept; Boston (MA).

20.Taylor BA, Webb PJ, Hetreed M, Mulukutla RD, Farrell J. Delayed postoperative paraplegia with hypotension in adult revision scoliosis surgery. Spine. 1994;19(4):470-4.

21.Winter RB. Neurologic safety in spinal deformity surgery. Spine.1997;22(13):1527-33.
22. Apel DM, Marrero G, King J, Tolo VT, Bassett GS. Avoiding paraplegia during anterior spinal surgery. The role of somatosensory evoked potential monitoring with temporary occlusion of segmental spinal arteries. Spine. 1991;16(8 Suppl):S365-70.

23.Pedrals JG. Spinal shortening in scoliosis surgery. A case with transitory paraplegia. Spine. 1996;21(21):2515-9.

24.Dove J. Internal fixation of the lumbar spine. The Hartshill rectangle. Clin Orthop Relat Res. 1986;(203):135-40.

25.Dove J. Luque segmental spinal instrumentation: the use of the Hartshill rectangle.Orthopedics. 1987;10(6):955-61.

\section{Correspondência}

Miguel Trandafilov Júnior

Av. Hélio Borenstein, 1275

Vila Oliveira - Mogi das Cruzes - SP

CEP: 08790-230

Tel: +55-11-4796-1002/

$11-8358-9949$

E-mail: migueltranda@hotmail.com 